ACTA UNIVERSITATIS LODZIENSIS

Folia Litteraria Romanica 15, 2020

https://doi.org/10.18778/1505-9065.15.20

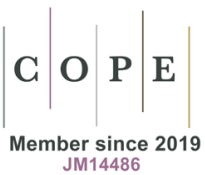

Anna Kaczmarek-Wiśniewska

Université d'Opole

iD ORCID : 0000-0002-8828-7039

akaczmarek@uni.opole.p1

\title{
Du « socialisme de cour » zolien, ou comment être considéré en même temps comme « socialiste » et « ennemi du peuple »
}

\begin{abstract}
RÉSUMÉ
Suite à la publication de Germinal en 1885, la critique a classé Zola comme un écrivain « socialiste ». Le romancier lui-même a utilisé ce qualificatif plusieurs fois en parlant de son œuvre. En même temps, il ignorait la doctrine marxiste et était un adversaire déclaré de la révolution, ainsi que de l'égalité et du gouvernement par le peuple. Le présent article se propose d'expliquer quelques aspects du « socialisme » de Zola compte tenu de ses origines, ses expériences de jeunesse et l'évolution de son œuvre, ce qui permettra de comprendre le sens particulier que l'écrivain donnait à ce terme.
\end{abstract}

MOTS-CLÉS - Zola, socialisme, peuple, question sociale, utopie

\section{'Zola's 'Socialism of the Heart', or How To Be Simultaneously a Socialist and an Enemy of The People"}

\section{SUMMARY}

When Germinal was published in 1885 , the critic classified Zola as a "socialist" writer. The author himself used this adjective many times referring to his work. At the same time, he didn't know Marxism and was a resolute opponent to revolution, equality and the government by the people. The paper aims to explain some aspects of Zola's "socialism" taking into account his origins, his youth experiences and the evolution of his works. This might help to understand the particular meaning done to the term by the writer.

KEYWORDS - Zola, socialism, people, social question, utopia

L'éternel recommencement de la misère, le travail de brute, ce destin de bétail $[\ldots]$, tout le malheur disparaissait $[\ldots]$; et $[\ldots]$ la justice descendait du ciel, $[\ldots]$ assur[ant] le bonheur des hommes [et] faisant régner l'égalité et la fraternité. Une 
société nouvelle poussait en un jour [...], une ville immense [...] où chaque citoyen vivait de sa tâche et prenait sa part des joies communes ${ }^{1}$.

Cette vision d'Étienne Lantier, dans Germinal (1885), traduit les idées de Zola sur la « question sociale » qui le préoccupe depuis sa jeunesse. En effet, avec ce roman, le treizième de la série des Rougon-Macquart, le romancier revient à un sujet qui n'était pas vraiment apparu dans son chef-d'œuvre de 1877, L'Assommoir : le conflit entre les ouvriers et les patrons; de ce fait, L'Assommoir " ne parlait pas exactement de la classe ouvrière », suite à quoi Zola « avait réussi son livre et raté son sujet $»^{2}$. Le « vaste mouvement socialiste qui travaille la vieille Europe d'une façon si redoutable $»^{3}$ pousse Zola à reparler des ouvriers, à étudier leur rôle politique ; Germinal devient ainsi « le complément de L'Assommoir, les deux faces de l'ouvrier $\gg{ }^{4}$.

En effet, Germinal, œuvre d'un auteur qui n'a jamais occupé de fonction politique, introduit vraiment et pleinement dans le roman - comme l'observe le critique marxiste André Wurmser - « cette classe ouvrière absente de La Comédie humaine », en le faisant « de façon plus authentique que ne l'avaient fait Victor Hugo ou Eugène Sue $»^{6}$. Chef-d'œuvre zolien le plus lu - malgré les craintes de son auteur, persuadé que le livre « fatiguera[it] le public » ${ }^{7}-$, il a valu à Zola l'étiquette de « socialiste ». Le romancier lui-même l'appelle son " roman socialiste ${ }^{8}$; dans des interviews et des articles datant de la fin des années 1880, il se déclare " profondément socialiste $»^{9}$ et qualifie de « socialiste » la totalité de son œuvre romanesque écrite jusque-là :

Je n'ai pas fait un livre sans parler de cette question [du socialisme], sans la faire intervenir dans le drame, dans l'étude, dans l'observation. [...] [J]e considère aujourd'hui plus que jamais que tout avenir est contenu dans ce mot de socialisme. C'est la pierre d'achoppement où viendra se heurter brusquement la société actuelle ${ }^{10}$.

1 É. Zola, Germinal, Paris, Fasquelle, 1982, p. 164.

2 A. Lanoux, Préface de Germinal, op. cit., p. IV.

3 É. Zola, lettre à J. Van Santen Kolff, 06/10/1889, in idem, Euvres complètes, édition établie sous la dir. D’H. Mitterand, t. XIV : Chroniques et Polémiques II, Tchou, Paris 1970, p. 1473. Les références à cette édition des œuvres de Zola seront désormais marquées dans le texte par l'abréviation O.C., suivie du numéro du volume et des pages correspondant à la citation.

4 Ibid.

5 A. Wurmser, « Les marxistes, Balzac, et Zola », Les Cahiers Naturalistes n 28, 1964, p. 138.

6 A. Barjonet, Zola d'Ouest en Est. Le Naturalisme en France et dans les deux Allemagnes, Rennes, Presses Universitaires de Rennes, 2010, p. 11.

7 É. Zola, lettre à H. Céard, 22/03/1885, in O.C., XIV, p. 1440.

8 H. Mitterand, Zola, t. II : L'homme de Germinal : 1871-1893, Paris, Fayard 2001, p. 734.

9 Zola socialiste. Une conversation avec l'auteur de « Germinal » et de «La Terre », « La Lanterne », 8 janvier 1887 , p. 1.

${ }^{10}$ É. Zola, fragment d'un article dans Le Gaulois du 25 avril 1890 ; cité d'après : H. Mitterand, Autodictionnaire Zola, Paris, Omnibus, 2012, p. 599. 
Plus encore que de pareilles proclamations, sa foi inébranlable en la nécessité de raser le vieil ordre et d'en installer un nouveau, assurant la justice et une vie digne aux classes déshéritées, semble le placer d'emblée parmi ceux qui chantent l'hymne de l'Internationale.

Zola est-il donc vraiment « socialiste »? La réponse à une question ainsi posée n'est pas facile ; le présent article, partant du fait que « les relations de Zola avec le socialisme sont à la fois constantes et complexes $\|^{11}$, se propose d'élucider quelques aspects de cette problématique.

\section{1. «Un bourgeois de gauche $»^{12}$ et son intérêt pour le peuple}

Si l'on admet que, au XIX ${ }^{\mathrm{e}}$ siècle, " chez un grand nombre de gens de lettres [...] le socialisme est un mot fourre-tout $»^{13}$, l'étiquette de « socialiste » attribuée à quelqu'un qui s'intéresse particulièrement à la vie des classes populaires n'est point infondée. En fait, Zola, républicain déclaré, a toujours été très sensible au sort de « ceux qui mangent leur pain dur et qui couchent sous les toits $\rangle^{14}$, si bien que la « question sociale» peut être considérée comme un leitmotiv de toute son œuvre ${ }^{15}$.

Par sa naissance, le romancier appartenait à la moyenne bourgeoisie. Du côté paternel, sa famille, originaire de Brescia en République vénitienne, représente une 《 petite bourgeoisie d'épée $~^{16}$, militaires de père en fils ; le père d'Émile, Francesco Zolla, qui s'installe définitivement en France et francise son nom, est titulaire d'un double diplôme d'ingénieur militaire et de docteur en mathématiques ${ }^{17}$. Quant aux Aubert, la famille de la mère d'Émile, Françoise-Émilie-Aurélie, ils sont d'une origine nettement plus modeste : Henriette Aubert, née Nogent, est couturière, et son mari Louis-Étienne un « artisan peu fortuné, vitrier comme son père [...] et peintre en bâtiment $»^{18}$. Fils des parents ambitieux qui considéraient que « [...] son adolescence doit être celle d'un jeune homme de bonne famille ${ }^{19}{ }^{19}$, le jeune Émile n'est pas censé connaître ceux qui deviendront un jour les personnages de ses romans ; sa mère, désireuse d' " assurer à son enfant un encadrement et une discipline serrés, et de qualité, sans pour autant faire appel aux établissements les

${ }^{11}$ S. Guermès, La Religion de Zola. Naturalisme et déchristianisation, Paris, Champion, 2006, p. 273.

${ }^{12}$ M. Girard, "Positions politiques d'Émile Zola jusqu'à l'affaire Dreyfus », Revue française de science politique $\mathrm{n}^{\circ} 3,1955$, p. 506.

${ }^{13}$ Ch. Charle, Naissance des « intellectuels », Paris, Éditions du Minuit, 1990, p. 104-105.

${ }^{14}$ É. Zola, Causerie [Les divertissements populaires], in O.C., XIII, p. 194.

${ }^{15}$ Cf. C. Becker, G. Gourdin-Servenière, V. Lavielle, Dictionnaire d'Émile Zola. Sa vie, son cuvre, son époque, Laffont, Paris, 1993, p. 344. Désormais dans les notes : Dictionnaire d'Émile Zola.

${ }^{16}$ H. Mitterand, Zola, t. I: Sous le regard d'Olympia. 1840-1871, Paris, Fayard, 1999, p. 18.

${ }^{17}$ M. Sementéry, «Au sujet de l'auteur de Germinal : les racines d'Émile Zola », Histoire et sociétés. Annales de généalogie et d'héraldique $\mathrm{n}^{\circ}$ 47, 1993, p. 12.

${ }_{18}$ Dictionnaire d'Émile Zola, p. 43.

${ }^{19}$ H. Mitterand, Zola, t. I, op. cit., p. 91. 
plus huppés - et les plus onéreux - de la ville $»^{20}$, l'envoie d'abord à une pension catholique, puis à un collège public laïque, faisant ainsi un choix « de fidélité à l'indépendance intellectuelle de son mari $»^{21}$.

La situation financière de la famille se dégrade après la mort prématurée de François Zola ; Émile connaîtra alors à fond la vie de ceux qu'il avait autrefois évités : au début de son séjour à Paris (1858-1863), il souffrira lui-même de la pauvreté et vivra à côté des gens du peuple, ajoutant ainsi du vraiment vécu à ce qu'il aura lu et entendu en la matière ${ }^{22}$. À cette époque, Zola semble assez indifférent à la politique, mais la situation des ouvriers - dont le nombre dans la France de la fin du Second Empire frôle les 3 millions ${ }^{23}$ - le préoccupe déjà beaucoup : « ce qui le touche [...], ce sont les conséquences de l'inégalité sociale : misères, souffrances, angoisses de la souillure indélébile qu'elles laissent sur l'âme $»^{24}$.

S'il n'est donc pas prédestiné à devenir « socialiste » de par sa naissance, il sera pourtant fortement influencé par sa situation d'homme sans ressources, par sa « vie de 'cheval de manège' - [...] petit employé à soixante francs par mois $»^{25}$. Ce bagage de jeunesse l'incitera à parler de la « question sociale » et lui fera bientôt choisir le peuple comme sujet de ses livres ; il achèvera aussi de former l'orientation politique générale du futur romancier, celle de gauche, à laquelle il « penche d'instinct $»^{26}$ depuis son adolescence :

[...] libéral sous l'Empire, républicain en 1870, centre gauche vers 1880, sympathisant radical à la fin du siècle...[...], il a épousé à peu près l'évolution de son temps [...] [L']idéal [zolien] a toujours été le "progrès social » [...] Un bourgeois de gauche : tel est le terme [...] qui s'applique le mieux à Émile Zola ${ }^{27}$.

\section{Un « ennemi du peuple»}

C'est en tant que bourgeois que le jeune écrivain attire sur sa tête les foudres de la critique. Lecteur attentif de la presse et bientôt journaliste lui-même, bien avant d'être proclamé ennemi des valeurs républicaines suite à son engagement en faveur de Dreyfus, il est accusé d'avoir « calomnié » le peuple avec ses deux romans ouvriers. La publication en feuilleton de L'Assommoir - « le premier roman sur le peuple qui

\footnotetext{
${ }^{20}$ Ibid., p. 70.

${ }^{21}$ Ibid., p. 97.

${ }^{22}$ Le Second Empire était riche en enquêtes sur les « classes ouvrières » au point que le sujet « se banalise ». Cf. J. Tulard (dir.), Dictionnaire du Second Empire, Paris, Fayard, 1995, p. 945.

${ }^{23}$ Cf. J. Tulard, op. cit., p. 945.

${ }^{24}$ C. Becker, « Républicain sous l’Empire », Les Cahiers naturalistes n 54, 1980, p. 8.

${ }^{25}$ Ibid.

${ }^{26} \mathrm{Ibid}$.

${ }^{27}$ M. Girard, op. cit., p. 506.
} 
ne mente pas et qui ait l'odeur du peuple $»^{28}$ - inspire à Octave Mirbeau ces paroles sévères : « M. Zola [...] s'est dit et se dit encore l'ami du peuple, et il le diffame [...] Toutes les monstruosités morales [...], M. Émile Zola les prête à l'ouvrier $»^{29}$. On dénonce le prétendu mépris « tout à fait bourgeois » de l'auteur pour le peuple, mépris qui a donné naissance à une œuvre « malsaine et ordurière », un « pamphlet ridicule dirigé contre les travailleurs $»^{30}$. Victor Hugo lui-même constate que « le livre est mauvais » et refuse à Zola « le droit de nudité sur la misère et le malheur $»^{31}$. Les réactions à Germinal, généralement plus complaisantes, ne manquent pas non plus d'opinions négatives ; même Le Figaro, auquel Zola a collaboré, publie un article défavorable à ce roman au «titre bizarre et peu compréhensible », dont certaines parties sont « inutilement grossi[ères] $»^{32}$. La critique de droite impute à l'écrivain une profonde méconnaissance de la vie des ouvriers, une vision « du dehors », décalée, menant à «l'idée la plus erronée $\mathrm{d}[\mathrm{u}][\ldots]$ travail $»^{33}$; on ignore des mois de lectures et de documentation, ainsi que la semaine du 23 février au 2 mars 1884 passée par l'auteur dans le pays des mines (y compris un voyage sous terre) $)^{34}$. À son tour, la critique de gauche accuse Zola d'avoir perpétué l'image de l'ouvrier ivrogne, débauché, fainéant et totalement incapable de prendre la responsabilité de son propre sort - donc parfaitement conforme à l'imaginaire bourgeois de l'époque.

Zola ne reste pas indifférent à ces dénigrements ; un des critiques hostiles à $L ' A s s o m m o i r$ reçoit la réponse suivante :

Vous me traitez d'écrivain démocratique et quelque peu socialiste [...]. - D'abord, je n'accepte pas l'étiquette que vous me collez dans le dos. J'entends être un romancier tout court, sans épithète ; si vous tenez à me qualifier, dites que je suis un romancier naturaliste [...]. Mes opinions politiques ne sont pas en cause [...]. Quant à ma peinture d'une certaine classe ouvrière, $[. .$.$] je dis ce que je vois, je verbalise simplement, et je laisse aux moralistes$ le soin de tirer la leçon. [...] Mon œuvre n'est pas une œuvre de parti et de propagande : elle est une œuvre de vérité35.

Et, dans une lettre ouverte au directeur du Figaro, il écrit :

[...] on m'accuse [...] de fantaisie ordurière et de mensonge prémédité sur de pauvres gens [...]. À chaque accusation, je pourrais répondre par un document. Pourquoi veut-on que je calomnie les misérables? Je n'ai eu qu'un désir, les montrer tels que notre société les fait $[\ldots]^{36}$.

${ }^{28}$ É. Zola, Préface de L'Assommoir, Paris, Fasquelle,1980, p. 8.

${ }^{29}$ O. Mirbeau, Combats littéraires, Paris, L'Âge d'Homme, 2006, p. 35.

${ }^{30}$ Propos du député Charles Floquet, 1879 ; cité d'après : M. Girard, op. cit., p. 516.

${ }^{31}$ H. Mitterand, Zola, t. II : L'Homme de Germinal, Paris, Fayard, 2001, p. 310.

${ }^{32}$ H. Duhamel, «Émile Zola et les mineurs », Le Figaro, 4/04/1885, p. 1.

${ }^{33}$ Ibid.

${ }^{34}$ A. Pagès, O Morgan, Guide Émile Zola, Paris, Ellipses, 2002, p. 507.

${ }^{35}$ É. Zola, lettre à A. Millaud, in Correspondance : les lettres et les arts, Paris, Fasquelle, 1908, p. 76.

${ }^{36}$ É. Zola, lettre à F. Magnard, 4/04/1885, in O.C., XIV, p. 1441. 
Loin de s'inscrire dans la lignée romantique de l'auteur des Misérables, le romancier, désireux de «ne pas noyer [les pauvres gens] sous des flots de compassion ou de pardon, mais les donner à voir et à entendre, prendre toute la mesure de leur condition et de ses fatalités ${ }^{37}$, tâche d'utiliser à ce dessein ce qu'il considère comme la méthode naturaliste : l'observation « clinique » et le diagnostic « scientifique ». La pensée positive, ainsi que le changement du climat social lié à la présence de plus en plus massive du peuple dans les villes, sont les clés de sa réflexion.

\section{Un socialiste sceptique envers le(s) socialisme(s)}

Au milieu des années 1880, Zola écrit : "Toutes les fois maintenant que j'entreprends une étude, je me heurte au socialisme $»{ }^{38}$. Il est parfaitement conscient de la montée, parmi les travailleurs, de la conscience politique et sociale qui les pousse à défendre leurs droits et à formuler des revendications adressées aux patrons, portant surtout sur la longueur de la journée du travail et le montant de la paie ; en effet, réprimés en France au temps de la Seconde République, les courants socialistes « réapparaissent au grand jour après l'amnistie des années 1879-80 »" Collaborateur aux journaux de l'opposition à l'Empire (Le Rappel, La Tribune, Le Voltaire), le romancier aura aussi l'occasion de suivre des histoires de litiges entre les patrons et les ouvriers et de se rendre compte du développement de l'esprit de résistance ouvrière, allumé aussi bien par « le sentiment de l'intolérable » que par «l'étincelle des idéologies révolutionnaires les plus diverses $»^{40}$. Évidemment, il ne manquera pas la création à Londres, le 4 novembre 1864, de l'Internationale dont les premières sections françaises ne tardent pas à apparaitre et qui passe bientôt de la théorie à la pratique, soutenant, en 1871, la Commune de Paris. Bref, « [i]l ne peut échapper à Zola que le mouvement ouvrier est en voie de réorganisation, [...] qu'il se dote d'une idéologie et d'une stratégie neuves, et que s'y manifeste une combativité croissante, surtout dans les régions minières $\gg{ }^{41}$.

En effet, l'auteur s'informe sur la vague des grèves qui secoue la France à la fin des années 1860 ; l'une d'elles, celle d'Anzin (1866), lui servira de modèle pour Germinal. Désireux de comprendre ce qui lui paraît encore confus, il assiste, le 7 mars 1884, à une réunion publique dont l'un des animateurs est Jules Guesde, fondateur de L'Égalité (premier journal socialiste inspiré des thèses de Marx) et un des chefs du Parti ouvrier; on y parle, entre autres, de la nouvelle grève

${ }^{37}$ H. Mitterand, Zola, t. II, op. cit., p. 313.

${ }^{38}$ É. Zola, lettre à J. Van Santen Kolff, 27/05/1886, in O.C., XIV, p. 1452.

${ }^{39}$ P. Ouvrard, «A propos de Germinal (1885) : Zola et le socialisme romantique », Revue d'Histoire littéraire de la France vol. 85, n 3, p. 427.

${ }^{40}$ H. Mitterand, Zola, t. II, op. cit., p. 715.

${ }^{41}$ Ibid., p. 717. 
d'Anzin. Voilà la fin de Germinal toute faite : Zola croit en savoir assez pour pouvoir commencer son roman.

Ayant gagné un savoir aussi riche et diversifié sur les classes populaires, Zola savait bien que la formule citée au début de cette partie de notre réflexion aurait dû mettre le nom « socialisme » au pluriel. Il s'est trouvé, en fait, devant différentes visions du socialisme qu'il a pu comparer et mesurer les différences qui les séparaient. Il s'est informé à leur propos en discutant avec ses amis (Paul Alexis, Jean Grave, Jean-Baptiste Noirot ou même Jean Jaurès) et en lisant de nombreux ouvrages (se contentant parfois, il faut le dire, des livres de seconde main $^{42}$ ) ; le Capital de Marx, dont la traduction française était accessible depuis $1875^{43}$, ne figure pas parmi ses lectures, ce qui le range parmi bon nombre de ses compatriotes de l'époque - même ceux qui se déclaraient socialistes -, qui méconnaissaient la théorie marxiste. Ce manque d'enthousiasme pour un des ouvrages-clés de la doctrine socialiste est peut-être dû à sa désapprobation "viscérale ${ }^{44}$ de toute doctrine, quelle que soit sa nature ; en 1887, le maître de Médan explique dans une interview :

De doctrines, je n'en ai pas, et j'avoue même qu'elles ne m'intéressent guère. Du reste, je $[\ldots]$ ne veux pas faire de politique. [...] Ce sont les faits, les aspirations de la foule vers un idéal de justice qui m'intéressent. Je m'occupe peu d'hier, et ce que sera demain me passionne ${ }^{45}$.

Même lorsque Paul Alexis ménage un entretien entre Zola et Jules Guesde, le 2 mai 1886, le résultat en est médiocre : « la grâce socialiste n'a pas touché Zola $»^{46}$ qui, ayant déclaré six ans avant, dans Le Roman expérimental : « Je ne tiens par aucune attache au monde politique, et n'attends du gouvernement ni place, ni pension, ni récompense d'aucune sorte $»^{47}$, se refuse toujours à tout engagement politique.

L'écrivain a analysé les théories socialistes progressivement; il les a analysées, a mesuré leur complexité, et, sceptique par nature et par son expérience, il est arrivé à la conclusion qu'il fallait rejeter le socialisme collectiviste d'inspiration marxiste et défendre une organisation de la société fondée non sur une révolution violente, mais sur la solidarité entre les classes sociales. Cette vision, bâtie pour l'essentiel sur les idées du socialiste utopique Charles Fourier, alimentera le roman Travail (1901).

${ }^{42}$ Cf. Dictionnaire d'Émile Zola, op. cit., p. 393-394.

${ }^{43}$ H. Mitterand, Zola, t. II, op. cit., p. 716.

${ }^{44}$ Cf. A. Barjonet, op. cit., p. 109.

${ }^{45}$ Zola socialiste. Une conversation, op. cit.

${ }^{46}$ M. Rebérioux, "Zola et la critique littéraire française socialiste et anarchiste », Europe n ${ }^{\circ}$ 468-469, 1968, p. 9.

${ }^{47}$ É. Zola, Le Roman expérimental, cité d'après : H. Mitterand, Autodictionnaire Zola, op. cit., p. 552-553. 


\section{Un républicain anti-révolutionnaire et anti-égalitariste}

La critique souligne avec justesse que les romans de Zola « ne présentent pas le marxisme, le socialisme et encore moins l'anarchisme sous un jour favorable » et que «sa galerie de personnages de gauche n'est pas flatteuse $[\ldots] »^{48}$. En effet, s'ils ne sont pas touchés, comme Étienne Lantier, par la « fêlure » héréditaire des Rougon-Macquart, ils sont pourtant tous inadaptés au monde qui les entoure, cette inadéquation prenant des formes diverses : le rêveur Busch (L'Argent) vit dans un univers utopique et consacre sa vie à inventer les bases théoriques de la société à venir ; le naïf Florent (Le Ventre de Paris) œuvre pour une révolution pacifique ; le révolutionnaire Leroi, dit Canon ( $L a$ Terre), incite les paysans à organiser euxmêmes leur bonheur; enfin, l'anarchiste Souvarine (Germinal) prêche la destruction totale de la société existante, n'hésitant pas à passer à l'action, coûte que coûte. L'écrivain semble donc garder ses distances à l'égard de ses personnages socialistes, surtout de ceux dont les paroles et les actions sont guidées par la violence et l'indifférence au sort des autres.

Une des causes de cette distanciation peut être la répugnance vouée par l'écrivain à toute forme violente de bouleversement social, à toute solution brutale qui pourrait provoquer la guerre civile et le chaos. Selon lui, « la révolution [...] est inquiétante parce qu'elle [...] évoque [...] des images de ruines, de violence, de sang, de meurtre. Elle est [...] débordement de la bestialité, des instincts, déferlement des barbares ${ }^{49}$. Si Zola reste parfaitement conscient de la nécessité de l'évolution sociale conforme aux prouesses de l'époque, il se heurte pourtant sans cesse contre la quasi impossibilité de concilier les droits de l'individu et ceux de la société. Confiant dans la « marche des temps », prônant la grande loi du progrès, le romancier finit par constater que l'avenir est dans la science : c'est grâce à ses conquêtes que pourra s'opérer une évolution des mentalités qui, à son tour, amènera l'établissement d'une société nouvelle, celle « du juste et du vrai », fondée sur le travail de tous. Or, s'il considère comme néfaste tout ce qui peut gêner l'avènement de cette société, il refuse pourtant catégoriquement la révolution comme moyen d'y parvenir.

Bien que les bases idéologiques de cette vision soient les valeurs telles que l'équilibre, la fraternité, la justice, la paix et la liberté50, exprimées par une action quotidienne, continuelle, acharnée de tous et de chacun, la devise de ce royaume des heureux n'est point celle de la révolution de 1789. En effet, « des trois termes de la trilogie révolutionnaire, 'Liberté, Égalité, Fraternité', [Zola] n'emploie pas le second $»^{51}$. Interviewé, en 1890, par un journaliste du New York Herald Tribune, il prononce ces paroles lourdes de sens : «[...] le monde a-t-il été rendu

\footnotetext{
${ }^{48}$ A. Barjonet, op. cit., p. 9.

${ }^{49}$ Dictionnaire d'Émile Zola, op. cit., p. 365-366.

${ }^{50}$ Cf. C. Becker, op. cit., p. 10.

${ }^{51}$ Ibid.
} 
meilleur par notre grande Révolution ? Les hommes sont-ils [...] en réalité plus égaux qu'ils ne l'étaient il y a cent ans ? [...] - Non ! Alors arrêtez de parler de l'égalité ! La liberté, oui ; la fraternité, oui ; mais l'égalité, jamais ! $»^{52}$. Persuadé que toute personne doit pouvoir développer sa personnalité et admirant les grandes individualités de l'époque, l'écrivain s'oppose vivement à la vision proudhonienne de la « ville modèle », bâtie sur l'« enrégimentement » des esprits suite auquel «l'homme n'est plus qu'un infime manœuvre $»^{53}$. Poursuivant son idéal d'harmonie universelle, il rêve, au contraire, d'une " cité idéale de justice et de liberté " $^{54}$ composée d'individus heureux et accomplis. C'est autour de ce rêve que seront conçus Les Quatre Évangiles, dernier cycle romanesque zolien.

\section{Un utopiste}

En 1880, dans une lettre au rédacteur du Figaro, Zola avertissait : "C'est un républicain qui entre au Figaro et qui vous demandera beaucoup d'indépendance personnelle pour y défendre ce qu'il croit être la vérité, aussi bien dans la politique que dans les lettres $»^{55}$. La hardiesse de cette constatation d'un homme qui connaît bien sa valeur ouvrait le chemin à ce qui s'est passé une quinzaine d'années plus tard : à la « vérité en marche » qui a conduit l'écrivain à plaider pour Dreyfus.

Or, le Zola-dreyfusard n'est plus tout à fait le tribun qu'il fut une décennie plus tôt. Il est de plus en plus effrayé par la direction dans laquelle la société évolue : le capitalisme triomphant et le socialisme révolutionnaire ; souhaitant un gouvernement pour le peuple, il n'a jamais accepté l'idée d'un gouvernement par le peuple ${ }^{56}$, surtout si celui-ci devait naître dans le sang. En effet, c'est avec une grande lucidité que l'auteur du Travail avertit ses lecteurs de la dérive totalitaire que pourrait connaître une république socialiste née d'une révolution sanglante : prévoyant l'arrivée de la terreur de "l'affreuse guerre civile » si jamais « les collectivistes » devenaient « les maîtres du pouvoir $»^{57}$, l'auteur du XIX siècle procède ainsi à une singulière anticipation de ce que sera, quelques décennies plus tard, le stalinisme en URSS.

Ainsi, Zola s'accroche toujours plus à son rêve d'une cité parfaite, libérée des entraves d'une religion morte, des conventions vides et des faux principes menant l'homme à l'avilissement. C'est en 1898, durant son exil forcé en Angleterre,

52 J.-F. Revel, La Grande Parade. Essai sur la survie de l'utopie socialiste, Paris, Plon, 2000, p. 255.

${ }^{53}$ É. Zola, Proudhon et Courbet, http://www.cahiers-naturalistes.com/pages/Proudhon.html ; consulté le 17.09.2019.

${ }^{54}$ Idem, Causerie, in O.C., XIII, p. 116.

${ }^{55}$ Idem, lettre à F. Magnard, 16/09/1880, in O.C., XIV, p. 1411.

${ }^{56}$ Cf. C. Becker, op. cit., p. 16.

${ }^{57}$ É. Zola, Travail, in idem, Euvres complètes, op. cit., t. VIII, p. 965-966. 
qu'il découvre le fouriérisme et est « ébloui » par la convergence entre ses propres idées et l'« harmonie universelle» de Fourier :

Un ami m'a prêté Fourier et je le lis en ce moment avec éblouissement. Je ne sais encore ce qui adviendra de mes recherches, mais je veux glorifier le travail et, par là, obliger les hommes qui le profanent, l'asservissent, le souillent de laideur et de misère, à le respecter enfin ${ }^{58}$.

En effet, Les Quatre Évangiles se présentent comme une vaste utopie, combinant le travail aisé, le bien-être matériel et le développement personnel sur tous les plans grâce à une réforme sociale réalisée en douceur. La cité de la Crêcherie, dans Travail, devient l'incarnation de l'idéal zolien de l'harmonie et de la fraternité, et la lumière électrique qui l'illumine figure le progrès de l'intelligence qui illumine l'humanité. À Jean Jaurès, venu le voir à Londres, Zola déclare : « Je veux donner tout mon effort à la libération des hommes $»^{59}$. Et à Octave Mirbeau (devenu entre-temps un ami) qui reproche à Zola un style « catéchistique » et trop de didactisme dans son œuvre, le romancier répond avec humour : « Tout cela est bien utopique, mais que voulez-vous ? Voici quarante ans que je dissèque, il faut permettre à mes vieux jours de rêver un peu $»^{60}$.

\section{Conclusion}

Qu'en est-il donc du « socialisme » de Zola?

Selon la critique, « [1] discours politique de Zola [...] n'est certes pas banal, mais il n'est pas non plus exceptionnel $»^{61}$. Il s'agit pour autant d'un discours bien curieux, du moins du point de vue de la doctrine : républicain, mais antirévolutionnaire, anti-égalitariste, méfiant par rapport aux divers mouvements socialistes, son auteur s'avère être un « socialiste " plutôt singulier.

Le discours de Zola reste pourtant parfaitement conforme à sa propre définition du socialisme : « [1] e vrai socialiste, écrit Zola, $[\ldots]$ est [...] celui qui dit la misère, les déchéances fatales du milieu, qui montre le bagne de la faim dans son horreur ! $[\ldots] »^{62}$. Le marxiste André Wurmser n'a donc point tort en constatant que, si on ne peut pas « célébrer en Zola le socialiste scientifique, le marxiste qu'il n'a pas été », il faut cependant honorer en lui « l'écrivain progressiste, le peintre des mineurs et des paysans, l'homme de progrès et de conscience $»^{63}$.

\footnotetext{
58 A. Morice, «Travail, roman de Zola, ou la « race » ouvrière entre malédiction et rédemption », Tumultes $\mathrm{n}^{\circ} 26$ (2006/1), p. 88.

${ }^{59}$ A. Wurmser, op. cit., p. 147.

${ }^{60}$ É. Zola, lettre à O. Mirbeau, 29/11/1899, in Correspondance : les lettres et les arts, op. cit., p. 230.

${ }^{61}$ J.-C. Cassaing, H. Mitterand, Avant-propos, Les Cahiers naturalistes n ${ }^{\circ}$ 54, 1980, p. 4.

${ }^{62}$ É. Zola, lettre à G. Montorgueil, 8/03/1885, in O.C., XIV, p. 1438.

${ }^{63}$ A. Wurmser, op. cit., p. 145.
} 
L'attitude de Zola par rapport au socialisme peut donc se résumer ainsi : il est un socialiste " plus de cœur que de raison ${ }^{64}$, et, dans cette logique, le socialisme est chez lui souvent abordé en des termes généraux. En effet, liberté, justice, fraternité, paix sont les grands mots porteurs qui semblent lui suffire pour exprimer ce qui, dans son esprit, devrait un jour triompher de la dépravation et de l'immoralité de la société de son temps. Et même si son discours ne paraît ni très concret ni absolument cohérent, le romancier reste toujours, dans ses idées " socialistes ", fidèle à son idéal le plus cher : l'émancipation de l'humanité.

\section{Bibliographie}

Barjonet, Aurélie, Zola d'Ouest en Est. Le Naturalisme en France et dans les deux Allemagnes, Rennes, Presses Universitaires de Rennes, 2010

Becker, Colette, Gourdin-Servenière Gina, Lavielle Véronique, Dictionnaire d'Émile Zola. Sa vie, son æuvre, son époque, Paris, Laffont, 1993

Becker, Colette, « Républicain sous l'Empire », Les Cahiers naturalistes n 54, 1980, p. 7-16

Cassaing, Jean-Claude, Mitterand Henri, Avant-propos, Les Cahiers naturalistes n ${ }^{\circ}$ 54, 1980, p. 3-4

Charle, Christophe, Naissance des « intellectuels », Paris, Éditions du Minuit, 1990, p. 104-105

Duhamel, Henri, «Émile Zola et les mineurs », Le Figaro, 4/04/1885, p. 1

Girard, Marcel, « Positions politiques d'Émile Zola jusqu'à l'affaire Dreyfus », Revue française de science politique, $\mathrm{n}^{\circ}$ 3, 1955, p. 503-528

Guermès, Sophie, La Religion de Zola. Naturalisme et déchristianisation, Paris, Champion, 2006

«Henri Mitterand présente le 'grand Zola' du xx siècle », Le Monde $\mathrm{n}^{\circ}$ 6872, 15 février 1967, p. V Lanoux, Armand, Préface à Germinal, Paris, Fasquelle, 1982, p. III-VI

Sementéry, Michel, «Au sujet de l'auteur de Germinal : les racines d'Émile Zola », Histoire et sociétés. Annales de généalogie et d'héraldique, no 47, 1993, p. 11-22

Mirbeau, Octave, Combats littéraires, Paris, L'Âge d'Homme, 2006

Mitterand, Henri, Autodictionnaire Zola, Paris, Omnibus, 2012

Mitterand, Henri, Zola, t. I : Sous le regard d'Olympia. 1840-1871, Paris, Fayard, 1999, t. II : L'homme de Germinal : 1871-1893, Paris, Fayard, 2001

Morice, Alain, « Travail, roman de Zola, ou la 'race' ouvrière entre malédiction et rédemption », Tumultes no $26(2006 / 1)$, p. 75-97

Ouvrard, Pierre, «A propos de Germinal (1885) : Zola et le socialisme romantique », Revue d'Histoire littéraire de la France vol. 85, no 3, p. 427-434.

Rebérioux, Madeleine, «Zola et la critique littéraire française socialiste et anarchiste », Europe $n^{\circ}$ 468-469, 1968, p. 7-15

Revel, Jean-François, La Grande Parade. Essai sur la survie de l'utopie socialiste, Paris, Plon, 2000 Tulard Jean (dir.), Dictionnaire du Second Empire, Paris, Fayard, 1995

Wurmser, André, « Les marxistes, Balzac, et Zola », Les Cahiers Naturalistes n 28, 1964, p. 137-148

Zola, Émile, Correspondance : les lettres et les arts, Paris, Fasquelle, 1908

Zola, Émile, Germinal, Paris, Fasquelle, 1982

Zola, Émile, Euvres complètes, édition établie sous la direction d'Henri Mitterand, t. XIII : Chroniques et Polémiques I, t. XIV : Chroniques et Polémiques II, Paris, Tchou, 1969-1970

${ }^{64} C f$. « Henri Mitterand présente le 'grand Zola' du xx ${ }^{\mathrm{e}}$ siècle », Le Monde $\mathrm{n}^{\mathrm{o}}$ 6872, 15 février 1967, p. V. 
Zola, Émile, Préface de L'Assommoir, Paris, Fasquelle 1980, p.7-8

Zola, Émile, Proudhon et Courbet, http://www.cahiers-naturalistes.com/pages/Proudhon.html ; consulté le 17/09/2019

Zola socialiste. Une conversation avec l'auteur de " Germinal » et de « La Terre », « La Lanterne », 8 janvier 1887 , p. 1

Anna Kaczmarek-Wiśniewska, docteur ès lettres HDR, est maître de conférences à l'Université d'Opole. Elle s'intéresse au roman et à la nouvelle réaliste et naturaliste, et tout particulièrement à l'œuvre romanesque et journalistique d'Émile Zola à laquelle elle a consacré deux livres et une quarantaine d'articles publiés en Pologne et à l'étranger (France, Canada, République tchèque, Slovaquie).

\begin{tabular}{|l|l|}
\hline cc Creative & $\begin{array}{l}\text { Co by the author, licensee Łódź University - Łódź University Press, } \\
\text { Łódź, Poland. This article is an open access article distributed under } \\
\text { the terms and conditions of the Creative Commons Attribution license } \\
\text { CC-BY-NC-ND 4.0 (https://creativecommons.org/licenses/by-nc-nd/4.0/) }\end{array}$ \\
\cline { 2 - 2 } & Received: 2019-01-25; Accepted: 2020-11-28 \\
\hline
\end{tabular}

